\title{
Manejo nutricional para pacientes hospitalizados com COVID-19: Uma revisão integrativa
}

\author{
Nutritional management for hospitalized patients with COVID-19: an integrative review \\ Manejo nutricional para pacientes hospitalizados con COVID-19: una revisión integradora
}

\begin{abstract}
RESUMO
O objetivo deste estudo é descrever a terapia nutricional adotada no cuidado aos pacientes hospitalizados com COVID-19. Este trabalho é uma revisão bibliográfica integrativa. Os dados foram buscados em bases de dados e portais. Verificou-se que os autores recomendam a realização da triagem de risco nutricional. Além disso, constatou-se que o ideal é o uso de terapia nutricional Enteral, utilizando-se uma dieta polimérica isosmótica padrão e uso de proteína entre 1,2-2,0 g/Kg/dia. A presença da Equipe Multidisciplinar em Terapia Nutricional é fundamental no tratamento e recuperação dos pacientes com COVID-19.
\end{abstract}

DESCRITORES: COVID-19; recomendações nutricionais; e terapia nutricional.

\section{ABSTRACT}

The aim of this study is to describe the nutritional therapy adopted in the care of patients hospitalized with COVID-19. This work is an integrative literature review. The data were searched in databases and portals. It was found that the authors recommend carrying out nutritional risk screening. In addition, it was found that the ideal is the use of Enteral nutritional therapy, using a standard isosmotic polymeric diet and use of protein between 1.2-2.0 g / Kg / day. The presence of the Multidisciplinary Team in Nutritional Therapy is essential in the treatment and recovery of patients with COVID-19.

DESCRIPTORS: COVID-19; nutritional recommendations; and nutritional therapy.

\section{RESUMEN}

El objetivo de este estudio es describir la terapia nutricional adoptada en el cuidado de los pacientes hospitalizados con COVID-19. Este trabajo es una revisión integradora de la literatura. Los datos se buscaron en bases de datos y portales. Se encontró que los autores recomiendan realizar un cribado de riesgo nutricional. Además, se encontró que lo ideal es el uso de terapia nutricional enteral, utilizando una dieta polimérica isosmótica estándar y uso de proteína entre 1.2-2.0 g/Kg/día. La presencia del Equipo Multidisciplinario en Terapia Nutricional es fundamental en el tratamiento y recuperación de los pacientes con COVID-19.

DESCRIPTORES: COVID-19; recomendaciones nutricionales; y terapia nutricional.

RECEBIDO EM: 30/09/2020 APROVADO EM: 14/10/2020

\section{Daniela Corrêa Ferreira}

Graduada em Nutrição pela Universidade Federal de Ouro Preto, especialista em Nutrição Clínica pela Universidade Veiga de Almeida. Mestre e Doutora em Ciência de Alimentos pela Universidade Federal de Minais Gerais. Professora Adjunta do Departamento do Curso de Nutrição da Universidade Federal de Juiz de Fora - Campus Governador Valadares.

ORCID: 0000-0002-1480-2974

\section{Deysimara Cássia Santos}

Discente do curso de Nutrição na Universidade Federal de Juiz de Fora, bolsista do Programa de Educação pelo Trabalho para a Saúde Pet/Saúde - Interprofissionalidade UFJF- GV/SMS-GV, monitora da disciplina Gestão de Unidade de Alimentação I e ligante da Liga Acadêmica Multiprofissional de Promoção de Saúde Infantil.

ORCID: 0000-0001-7472-9222 


\section{Maisa Pereira Vieira}

Acadêmica de Nutrição na Universidade Federal de Juiz de Fora, componente do grupo de pesquisa Núcleo de Estudos da Pessoa Idosa. É extensionista no projeto Ação, Cidadania, Juventude e Comunidade - Turmalina (Programa Boa Vizinhança - Rio Doce) é também ligante da Liga Acadêmica Multiprofissional de Promoção de Saúde Infantil, além de ser bolsista no projeto de Extensão Estratégias de intervenção para o envelhecimento ativo na comunidade - Grupo operativo de apoio ao emagrecimento sustentável e alimentação.

ORCID: 0000-0003-1127-9353

\section{Natália da Cunha Severino Sampaio}

Possui graduação em Enfermagem pela Pontifícia Universidade Católica de Minas Gerais. Pós-graduação em Enfermagem em Terapia Intensiva pela Pontifícia Universidade Católica de Minas Gerais. Pós-graduação em Enfermagem em Terapia Intensiva pela Pontifícia Universidade Católica de Minas Gerais. Atualmente é enfermeira da EMTN e da Equipe de Cuidados Paliativos e membro do Núcleo de Segurança do Paciente do Hospital Eduardo de Menezes (FHEMIG).

ORCID: 0000-0002-5799-8006

\section{Simonton Silveira de Andrade}

Possui graduação em Nutrição pela Universidade Federal e Ouro Preto. Mestrado em Ciências Biológicas (Fisiologia e Farmacologia) pela Universidade Federal de Minas Gerais e Doutorado em Ciências Biológicas (Fisiologia) pela Universidade Federal de Minas Gerais. Professor Associado do Departamento de Nutrição da Universidade Federal de Juiz de Fora - Campus Governador Valadares.

ORCID: 0000-0003-3177-9611

\section{INTRODUÇÃO}

A COVID-19 é uma doença causada por uma nova cepa de um vírus da família Coronaviridae ${ }^{1}$ conhecida como síndrome respiratória pandêmica associada ao novo coronavírus (SARS-CoV-2), cuja principais formas de transmissão são gotículas e aerossóis ${ }^{2}$. Geralmente, os pacientes desenvolvem doenças respiratórias com sinais e sintomas como: tosse, secreções nasais, dispneia, febre, mialgia e, em casos mais graves, dificuldade para respirar após o período médio de incubação de três a sete dias (variação de 2 a 14 dias) $)^{3}$.

A doença pode ser classificada em diferentes estágios: estágio 1 , em que o vírus infecta o hospedeiro, se multiplica e começa a afetar os pulmões, sendo caracterizada por sinais clínicos como: febre, pneumonia, tosse seca e dor de cabeça, podendo ainda levar a alterações hematológicas, neurológicas, gastrointestinais e metabólicas, principalmente em pacientes graves. O Centro de Controle de Prevenção de Doenças relata sintomas comuns como anosmia e disgeusia ${ }^{4}$. O estágio 2 é caracterizado por sintomas clínicos de falta de ar, hipóxia e sinais de imagem torácica anormal. Já, o estágio 3 é caracterizado por sintomas clínicos da síndrome do des- conforto respiratório agudo, síndrome da resposta inflamatória sistêmica, choque, insuficiência cardíaca ou disfunção de múltiplos órgãos, sendo ambos marcados por hiperinflamação, tempestade de citocinas e morte se não controlada ${ }^{3}$.

Estima-se que aproximadamente 20\% dos pacientes infectados podem apresentar sintomas graves o suficiente para requerer hospitalização, desses, $5 \%$ em estado crítico e necessitando da unidade de terapia intensiva (UTI), sendo que a maioria (75\%) exigirá ventilação mecânica ${ }^{2}$. A identificação precoce e o tratamento oportuno dos casos críticos de COVID-19 são de importância crucial, dado que ainda não há medicamentos com eficácia comprovada que tenham como alvo específico o SARS-CoV-25.

Estudos recentes destacaram a influência da COVID-19 no estado nutricional e na ingestão de nutrientes em pacientes infectados, além disso, quando comparados a pessoas saudáveis, verifica-se que eles apresentam aumento no gasto energético e maior risco de desnutrição. Diante disso, a não oferta adequada do aporte nutricional pode aumentar o tempo de internação hospitalar e a incidência de complicaçôes. Entretanto, a superalimentação tem sido associada a complicações como hiperglicemia, hipertrigliceridemia, esteatose hepática e aumento da taxa de mortalidade ${ }^{3}$.
Portanto, a oferta adequada de nutrientes merece destaque e deve ser observada para minimizar efeitos deletérios à saúde.

$\mathrm{O}$ estado nutricional pode ser comprometido pelo SARS-CoV-2, assim, pacientes hospitalizados que foram contaminados com o vírus, tendem a apresentar risco nutricional no momento da internação, visto que apresentam aumento do gasto energético. Dessa forma, a manutenção do estado nutricional é um componente integral das medidas no manejo de doenças infecciosas ${ }^{7}$. Portanto, o objetivo deste estudo é descrever a terapia nutricional adotada no cuidado aos pacientes hospitalizados com COVID-19.

\section{METODOLOGIA}

O presente estudo trata-se de uma revisão bibliográfica integrativa. Utilizou-se como estratégia de busca o método PVO, população, variáveis e desfecho, respectivamente. Logo, P são os pacientes com COVID-19, V a conduta nutricional adotada e $\mathrm{O}$, a manutenção e/ou recuperação do estado nutricional. Desta forma, buscou-se estudar os fatores associados à conduta nutricional em pacientes hospitalizados com COVID-19, ressaltando-se a importância da escolha do melhor plano terapêutico por meio da terapia nutricional. 
O levantamento bibliográfico foi realizado pela internet a partir das bases de dados e portais: LILACS, SciELO, ClinicalTrials.gov, PubMed, CAPES periódicos e Biblioteca virtual de saúde. Os descritores utilizados para a busca dos artigos foram: COVID-19; Recomendações nutricionais e Terapia Nutricional, bem como seus respectivos sinônimos em inglês. Os critérios de inclusão adotados basearam-se em artigos publicados nos últimos 8 meses, presença de pelo menos um dos descritores citados e estudos realizados em adultos. Foram excluídos os artigos de revisão e que não atendiam aos critérios acima citados.

Inicialmente, foram selecionados 24 artigos e após a análise, um total de 12 artigos foram utilizados para elucidação das recomendações de terapia nutricional em pacientes hospitalizados com COVID-19.

\section{RESULTADOS}

Os pacientes com COVID-19 devem ser assistidos por uma equipe multidisciplinar composta por médicos, enfermeiros, nutricionistas, farmacêuticos, fisioterapeutas e fonoaudiólogos, ressalta-se, que outros profissionais são necessários no apoio aos familiares. Além disso, evidenciou-se que, 69,2\% dos autores realizaram e recomendam que seja feita a triagem de risco nutricional dos pacientes com $\mathrm{CO}$ VID-19, sendo o instrumento mais utilizado (50\%) o Nutritional Risk Screening (NRS-2002), seguido pela Nutrition Risk in the Critical Ill (NUTRIC) modificada (33,3\%). O NUTRIC modificado foi escolhido em detrimento de sua versão ori-

\section{Os pacientes com \\ COVID-19 devem}

ser assistidos

por uma equipe

multidisciplinar

composta por

médicos, enfermeiros,

nutricionistas,

farmacêuticos,

fisioterapeutas e

fonoaudiólogos,

ressalta-se, que outros

profissionais são

necessários no apoio

aos familiares.

ginal, devido à dificuldade de obtenção da IL-6 na prática clínica 7 . Alguns artigos recomendaram a utilização de outras ferra- mentas complementares à triagem como: a Avaliação Subjetiva Global (16,7\%) e o Global Leadership Initiative on Malnutrition (16,7\%).

Em relação à conduta nutricional, $83,3 \%$ dos autores recomendaram o uso de terapia nutricional Enteral (TNE), iniciada em um período de 24-36h $(16,7 \%)$ ou $24-48 \mathrm{~h}(25 \%)$ após a admissão em UTI por meio de sonda nasogástrica em posição pós pilórica (75\%) com tubo de 10-12 french (16,7\%). Nos casos onde houve a necessidade de ventilação mecânica, recomendou-se o início da TNE em até $12 \mathrm{~h}$ após a intubação. Os demais estudos recomendaram o uso de suplementação oral $(8,3 \%)$ após a admissão hospitalar com $20 \mathrm{~g} /$ dia de proteína do soro de leite, seguido por nutrição parenteral em posição central $(8,3 \%)$ por um período de 18-24 h/dia após piora respiratória (pré-UTI). 16,7\% dos estudos indicaram o uso do método de cinco etapas: dieta + educação nutricional, suplemento nutricional oral, nutrição enteral (NE), nutrição parenteral suplementar e nutrição parenteral total.

Apenas 16,7\% dos estudos definiram a composição da dieta utilizada, adotando-se dieta polimérica isosmótica padrão. Além disso, 33,3\% dos estudos indicaram o uso de dietas hipocalóricas (15-20 kcal/ $\mathrm{Kg} / \mathrm{dia}$ ) e uso de proteína entre 1,2-2,0 g/ $\mathrm{Kg} / \mathrm{dia}(41,6 \%)$. Os demais recomendaram dietas normocalóricas $(41,6 \%)$ ou estimativa das necessidades energéticas através da fórmula de Harris-Benedict (8,3\%) e proteína entre 1,0-1,5 g/Kg/dia (33,3\%). Os resultados estão ilustrados na tabela 1 .

Tabela 1 - Características dos estudos sobre terapia nutricional na COVID 19.

$\begin{array}{ccc}\begin{array}{c}\text { Instrumento } \\ \text { de triagem }\end{array} & \text { Dieta } & \begin{array}{c}\text { Início da terapia } \\ \text { 24-36h após } \\ \text { admissão em UTI } \\ \text { ou até } 12 \text { h após a } \\ \text { intubação }\end{array} \\ \begin{array}{c}\text { 24-48h após a ad- } \\ \text { NRS-2002 / } \\ \text { NUTRIC } \\ \text { modificada }\end{array} & \text { NE } & \begin{array}{c}\text { missão em UTI ou o } \\ \text { mais precocemente } \\ \text { após a intubação. }\end{array}\end{array}$

\section{Fórmula da dieta}

Polimérica isosmótica padrão, progredindo para fórmula de fibra mista.

Conduta nutri-
cional
$15-20 \mathrm{kcal} / \mathrm{kg} /$ dia 1,2-2,0g pt/ $\mathrm{kg} / \mathrm{dia}$
$25-30 \mathrm{kcal} / \mathrm{kg} /$ dia e 1,2-2,0g $\mathrm{pt} / \mathrm{kg} / \mathrm{dia}$

$\begin{array}{ccc}\begin{array}{c}\text { Posição/ } \\ \text { sonda }\end{array} & \text { Infusão } & \text { Autor } \\ \begin{array}{c}\text { Nasogástrica/ } \\ \text { Pós pilórica }\end{array} & \begin{array}{c}\text { Bomba de } \\ \text { infusão } \\ \text { contínua }\end{array} & \begin{array}{c}\text { Patel et } \\ \text { al., } 2020 .\end{array} \\ \begin{array}{c}\text { Nasogástrica/ } \\ \text { Pós pilórica }\end{array} & - & \begin{array}{c}\text { Shang et } \\ \text { al. } 2020 .\end{array}\end{array}$




\section{artigo}

Ferreira, D.C.; Santos, D.C.; Vieira, M.P.; Sampaio, N.C.S.; Andrade, S.S.

Manejo nutricional para pacientes hospitalizados com COVID-19: Uma revisão integrativa

\begin{tabular}{|c|c|c|c|c|c|c|c|}
\hline - & NE & $\begin{array}{l}\text { 24-36h após a } \\
\text { admissão na UTI } \\
\text { ou em de } 12 \text { horas } \\
\text { após a intubação. }\end{array}$ & $\begin{array}{c}\text { Fórmula enteral iso- } \\
\text {-osmótica polimérica } \\
\text { hiperproteica ( } \geq 20 \% \\
\text { de proteína) na fase } \\
\text { aguda. }\end{array}$ & $\begin{array}{c}\text { 15-20kcal /kg/ } \\
\text { dia 1,2-2g pt/ } \\
\text { kg / dia }\end{array}$ & $\begin{array}{l}\text { Orogástrica } \\
\text { ou Nasogás- } \\
\text { trica }\end{array}$ & $\begin{array}{l}\text { Bomba de } \\
\text { infusão } \\
\text { contínua }\end{array}$ & $\begin{array}{l}\text { Martin- } \\
\text { dale, et al. } \\
2020 .\end{array}$ \\
\hline NRS-2002 & NE & - & - & $\begin{array}{c}\text { 27-30kcal/kg/ } \\
\text { dia e 1-1,5g pt / } \\
\text { Kg/dia }\end{array}$ & Nasogástrica & - & $\begin{array}{l}\text { Bruglie- } \\
\text { ra et al. } \\
2020 .\end{array}$ \\
\hline NRS-2002 & SO & - & - & $\begin{array}{c}\text { Utilizar equação } \\
\text { de Harris-Be- } \\
\text { nedict }\end{array}$ & $\begin{array}{l}\text { Infusão } \\
\text { central (se } \\
\text { disponível) ou } \\
\text { periférica }\end{array}$ & - & $\begin{array}{l}\text { Caccialan- } \\
\text { za et al., } \\
2020 .\end{array}$ \\
\hline & - & - & - & $\begin{array}{l}\text { dieta + educa- } \\
\text { ção nutricional, } \\
\text { ONS, alimenta- } \\
\text { ção por sonda, } \\
\text { NPS e NPT. }\end{array}$ & - & - & $\begin{array}{l}\text { Laviano et } \\
\text { al., } 2020 .\end{array}$ \\
\hline $\begin{array}{l}\text { NRS - } 2002 \\
\text { ou NUTRIC } \\
\text { modificada }\end{array}$ & NE & $\begin{array}{l}\text { 24-48h após a } \\
\text { admissão em UTI } \\
\text { ou após intubação }\end{array}$ & - & $\begin{array}{c}\text { Meta de } 30 \\
\text { kcal/kg/dia - } \\
\text { Iniciar com die- } \\
\text { ta hipocalórica, } \\
\text { e progredir até } \\
\text { hipercalórica e } \\
\text { 1,3-1,5g pt/kg } \\
\text { /dia }\end{array}$ & - & - & $\begin{array}{l}\text { Gupta et } \\
\text { al. } 2020 .\end{array}$ \\
\hline $\begin{array}{l}\text { NRS- } \\
\text { 200, ASG, } \\
\text { MAN,NUTRIC } \\
\text { e GLIM }\end{array}$ & $\begin{array}{l}\text { NE, se não } \\
\text { atender, } \\
\text { iniciar NP }\end{array}$ & - & - & $\begin{array}{l}\text { Calorimetria in- } \\
\text { direta ou dieta } \\
\text { hipocalórica } 20 \\
\text { kcal/kg/dia 1,3g } \\
\text { pt/kg dia }\end{array}$ & $\begin{array}{l}\text { Nasogástrica/ } \\
\text { Pós pilórica }\end{array}$ & - & $\begin{array}{l}\text { Barazzoni, } \\
\text { et al. } 2020\end{array}$ \\
\hline $\begin{array}{c}\text { NRS-2002 } \\
\text { NUTRIC }\end{array}$ & NE & $24-48 h$ & - & $\begin{array}{c}20-30 \mathrm{Kcal} / \mathrm{Kg} / \\
\text { dia 1,2-2,0g pt/ } \\
\text { Kg/dia }\end{array}$ & $\begin{array}{l}\text { Nasogástrica/ } \\
\text { Pós pilórica }\end{array}$ & - & $\begin{array}{l}\text { Qiu Haibo } \\
\text { et al., } \\
2020 .\end{array}$ \\
\hline- & NE & - & - & - & $\begin{array}{l}\text { Nasogástrica } \\
\text { ou Nasoje- } \\
\text { junal }\end{array}$ & $\begin{array}{c}\text { Bomba de } \\
\text { alimentação } \\
\text { enteral }\end{array}$ & $\begin{array}{l}\text { Anderson } \\
\text { et al., } \\
2020 .\end{array}$ \\
\hline SGA ou GLIM & & - & & $\begin{array}{l}\text { Dieta + educa- } \\
\text { ção nutricio- } \\
\text { nal20-30 Kcal/ } \\
\text { Kg/dia 1,0-2,0g } \\
\text { pt/ Kg/dia }\end{array}$ & - & - & $\begin{array}{l}\text { Yu Kaiying } \\
\text { et al., } \\
2020 .\end{array}$ \\
\hline $\begin{array}{l}\text { DEVE, MST, } \\
\text { MAN, [MNA- } \\
\text {-SF] }\end{array}$ & NE & $\begin{array}{l}\text { Até 24h após } \\
\text { admissão }\end{array}$ & - & $\begin{array}{l}25 \text { a } 30 \text { Kcal/ } \\
\text { kg/dia e 1,2g/ } \\
\text { ptn/kg/dia }\end{array}$ & $\begin{array}{l}\text { Nasogástrica } \\
\text { ou Pós-piló- } \\
\text { rica }\end{array}$ & - & $\begin{array}{l}\text { Chapple, } \\
\text { et al.2020 }\end{array}$ \\
\hline
\end{tabular}

\section{DISCUSSÃO}

A terapia nutricional é um componente integral das medidas de cuidados e suporte às doenças críticas. A doença possui fases e inclui uma fase aguda inicial, a fase pós-aguda imediata e o período de recuperação ${ }^{6}$. A fase aguda é dominada por um estado hipercatabólico no qual os aminoácidos são mobilizados como substrato para proteínas de fase aguda e produtos do sistema imunológico. É incontestável que a deterioração do estado nutricional interfere na evolução clínica do paciente, aumentando a morbimortalidade, o custo da hospitalização e piora da qualidade de vida ${ }^{19}$.
É notável a recomendação referente à realização da triagem do risco nutricional (69\%), reconhecida pelo Ministério da Saúde, a qual objetiva identificar o risco nutricional, garantindo uma avaliação rápida e de qualidade, direcionando a equipe de terapia nutricional ${ }^{20 ; 19}$. A prevenção, o diagnóstico precoce e o trata- 
mento da desnutrição, devem ser incluídos regularmente no manejo de pacientes com COVID-1911;21.

A NRS 2002 foi o instrumento mais utilizado (50\%) para realização da triagem de risco. Segundo a literatura, quando comparado com diferentes ferramentas de triagem nutricional, o NRS-2002 mostrou ser superior na identificação do risco de desnutrição durante a doença aguda ${ }^{22}$. Além disso, este é um método baseado em indicações de terapia nutricional relacionadas ao estado nutricional e necessidades nutricionais aumentadas diante da doença instalada ${ }^{20}$. Precedido pelo NRS 2002, o NUTRIC modificado foi o segundo instrumento mais utilizado, já que este foi desenvolvido especificamente para pacientes de UTI, assim, sendo mais específico para identificação do risco nutricional nesses pacientes $^{23}$. O critério adotado para optar pela NRS2002 ou NUTRIC deverá ser a gravidade do paciente ${ }^{24}$, sendo que o segundo é mais recomendado para pacientes graves ${ }^{23}$. Cabe ressaltar ainda, que para pacientes idosos o método MAN apresenta melhor especificidade e sensibilidade ${ }^{25}$.

Em relação à conduta nutricional, 83,3\% dos autores recomendaram o uso de TNE, iniciada em um período de $24-48 \mathrm{~h}$ (25\%) ou 24-36h (16,7\%) após a admissão em UTI. $\mathrm{O}$ paciente em terapia intensiva, frequentemente, encontra-se em estado hipercatabólico, decorrente do trauma, sepse ou de qualquer outro quadro de gravidade, assim o suporte nutricional para tais pacientes pode ser decisivo em sua evolução, pois a TN procura prevenir a deterioração do estado nutricional, além de minimizar as complicações devido ao jejum prolongado ${ }^{23}$.

Segundo o parecer da Sociedade Brasileira de Nutrição Parenteral e Enteral, a alimentação por via oral é a preferencial em pacientes não graves com diagnóstico de COVID-19, incluindo a utilização de suplementos orais quando a ingestão energética estimada for $<60 \%$ das necessidades nutricionais ${ }^{26}$. Porém, em pacientes graves, a $\mathrm{NE}$ é a via preferencial e sugere-se, que seja iniciada entre 24-48 horas, o que também corrobora com as diretrizes europeias e americanas de medicina intensiva ${ }^{27: 28: 29: 30}$. ...pacientes em estado grave e com estado nutricional comprometido, devem ser avaliados

\section{conforme as}

recomendações para

pacientes críticos,

uma vez que seu

estado nutricional

se assemelha a

pacientes com

doenças respiratórias

graves. Desta forma,

a evolução na oferta

das necessidades

energéticas deve

ocorrer lentamente,

visando à segurança

do paciente e

evitando uma

possível síndrome de

realimentação.
Em relação à recomendação energética, $33,3 \%$ dos estudos indicaram o uso de dietas hipocalóricas $(15-20 \mathrm{Kcal} / \mathrm{Kg} /$ dia $)$ e uso de proteína entre 1,2-2,0 g/Kg/dia (41,6\%). Os demais recomendaram dietas normocalóricas $(41,6 \%)$ ou estimativa das necessidades energéticas através da fórmula de Harris-Benedict (8,3\%) e proteína entre 1,0-1,5 g/ $\mathrm{Kg} / \mathrm{dia}(33,3 \%)$. Devido ao estado hipercatabólico desses pacientes e o risco de atrofia muscular 14, são indicados maiores teores de proteínas, principalmente, contendo aminoácidos de cadeia ramificada para promover maior síntese protéica?.

Em pacientes hospitalizados no início da doença que apresentam estado nutricional adequado, recomenda-se a introdução de dietas hipocalóricas nas primeiras semanas de internação ${ }^{31}$, evitando-se uma superalimentação. Todavia, pacientes em estado grave e com estado nutricional comprometido, devem ser avaliados conforme as recomendações para pacientes críticos, uma vez que seu estado nutricional se assemelha a pacientes com doenças respiratórias graves. Desta forma, a evolução na oferta das necessidades energéticas deve ocorrer lentamente, visando à segurança do paciente e evitando uma possível síndrome de realimentação ${ }^{6}$.

\section{CONCLUSÃO}

Conclui-se que a participação da Equipe Multidisciplinar em Terapia Nutricional é fundamental no tratamento e recuperação dos pacientes com COVID-19. A identificação do risco nutricional nas primeiras horas de internação e a intervenção nutricional assertiva, contribuem diretamente no desfecho favorável dessa doença pandêmica. Isso se justifica, uma vez que a doença pode ser deletéria ao estado nutricional dos pacientes, além de poder aumentar o tempo de permanência hospitalar, o risco de complicações e os gastos em saúde. Além disso, ressalta-se que aqueles pacientes que necessitam de respiração mecânica, são impossibilitados de alimentar-se por via oral, demandando nutrição enteral/parenteral, outrossim algumas situações de desospitalização poderão requerer assistência, para o restabelecimento por completo do estado de saúde.. 


\section{REFERÊNCIAS}

1. Gorbalenya, A. E. et al. The species Severe acute respiratory syndrome-related coronavirus: classifying 2019-nCoV and naming it SARS-CoV-2. Nature Microbiology, [S. I.], v. 5, n. 4, p. 536-544, 2020.

2. Patel, J. J.; Martindale, R. G.; Mcclave, S. A. Relevant Nutrition Therapy in COVID-19 and the Constraints On Its Delivery by a Unique Disease Process. v. 0, n. 0, 2020.

3. Gupta, L.; Jalan'o, G. A.; Gupta, P. Nutritional management and support in COVID-19: Emerging nutrivigilance. JPMA. The Journal of the Pakistan Medical Association, v. 70 3), n. 5, p. S124-S130, 2020.

4. Samaranayake, L. P.; Fakhruddin, K. S.; Panduwauala, C. Início súbito, perda aguda do paladar e do olfato na doença coronavírus 2019 (COVID-19): uma revisão sistemática. Acta Odontologica Scandinavica, v. 78, n. 6, pág. 467-473, 2020.

5. Li et al. Therapeutic strategies for critically ill patients with COVID-19. Ann. Intensive Care, v. 10, n. 45, 2020.

6. Fernández-Quintela, A. et al. Key Aspects in Nutritional Management of COVID-19 Patients. Journal of Clinical Medicine, v. 9, n. 8, p. 2589, 2020.

7. Oliveira, M. L. et al. Complementariedade do escore NUTRIC modificado com ou sem proteína C-reativa e avaliação subjetiva global na predição de mortalidade em pacientes críticos. Revista brasileira de terapia intensiva: São Paulo, v. 31, n. 4, Jan 20, 2020.

8. Laviano, A.; Koverech, A.; Zanetti, M. Nutrition support in the time of SARS-CoV-2 (COVID-19). Nutrition Elsevier Inc., 1 Jun. 2020.

9. Shang, Y.; Pan, C.; YANG, X. et al. Management of critically ill patients with COVID-19 in ICU: statement from front-line intensive care experts in Wuhan, China. Ann. Intensive Care, V. 10, N. 73, 2020.

10. Martindale, R. et al. Nutrition Therapy in Critically III Patients With Coronavirus Disease 2019. Journal of Parenteral and Enteral Nutrition, v. 0, n.0, 2020.

11. Brugliera, L. et al. Nutritional management of COVID-19 patients in a rehabilitation unit. European Journal of Clinical Nutrition, v. 74, n. 6, p. 860-863, 1 Jun. 2020.

12. Caccialanza, R. et al. Early nutritional supplementation in non-critically ill patients hospitalized for the 2019 novel coronavirus disease (COVID-19): Rationale and feasibility of a shared pragmatic protocol. Nutrition, v. 74, 2020.

13. Barazzoni, R. et al. ESPEN expert statements and practical guidance for nutritional management of individuals with SARS-CoV-2 infection. 2020.

14. Qiu Haibo, Li Xuyan, Du Bin, et al. Pensando no tratamento de nova pneumonia por coronavírus criticamente grave (1) [J / OL]. Chinese Journal of Tuberculosis and Respiratory, 2020, 43 (202002-23).

15. Anderson, L. Providing nutritional support for the patient with COVID-19. British Journal of Nursing, v. 29, n. 8, 2020.

16. Yu Kaiying, Shi Hanping. Interpretação de "Recomendações de especialistas sobre terapia nutricional médica para pacientes com pneumonia por Coronavírus Nova" [J]. Chinese Medical Journal, 2020,100 (10): 724-728. DOI: 10.3760 / cma.j .cn11213720200205-00196

17. Chapple, L. S. et al. Nutrition management for critically and acutely unwell hospitalised patients with coronavirus disease 2019 (COVID-19) in Australia and New Ze. Australian Critical Care, v. 33, pág. 399-406, 2020.

18. Sanches, F. L. F. Z.; Ferreira, T.; Guimarães, R. C. A. Risco nutricional em pacientes hospitalizados: comparação de três protocolos de triagem nutricional. Multitemas, v. 23, n. 55, p. 245, 2018.

19. Arruda, N. R.; Oliveira, A. C. C.; Garcia, L. J. C. Risco nutricional em idosos: comparação de métodos de triagem nutricional em hospital público. RASBRAN - Revista da Associação Brasileira de Nutrição. São Paulo, SP, Ano 10, n. 1, p. 59-65, Jan-Jun. 2019 - ISSN 23577894.

20. Sanson, G. et al. Prediction of early- and long-term mortality in adult patients acutely admitted to internal medicine: NRS-2002 and beyond. Clinical Nutrition, v. 39, n. 4, p. 1092-1100, 2020.

21. Pironi L. et al. Malnutrition and nutritional therapy in patients with SARS-CoV-2 disease. Clin Nutr. v. 20, 2020.

22. Reis, A. M.; Fructhenicht, A. V.; Moreira, L. F. NUTRIC score use around the world: a systematic review. Revista Brasileira de Terapia Intensiva: Porto Alegra, v. 31, n. 3, pág. 379-385, 2019.

23. Ferreira, C. J. F. et al. Nutritional therapeutic for the critically ill patients. Medicina, v. 36, n. 2-4, p. 394-398, 2003.

24. Barbosa, A. O.; Vicentini, A. P.; Langa, F. R. Comparação dos critérios da nrs-2002 com o risco nutricional em pacientes hospitalizados. Ciência e Saúde Coletiva: Rio de Janeiro, v. 24, n. 9, Set. 2019.

25. Raslan, M. et al. Aplicabilidade dos métodos de triagem nutricional no paciente hospitalizado. Revista de Nutrição: Campinas, v. 21, n. 5, 2008.

26. Campos, L. F. et al. Parecer BRASPEN/ AMIB para o Enfrentamento do COVID-19 em Pacientes Hospitalizados. BRASPEN, 2020.

27. Mcclave, S. A. et al. Diretrizes para o fornecimento e avaliação da terapia de suporte nutricional em pacientes adultos em estado crítico: Society of Critical Care Medicine (SCCM) e American Society for Parenteral and Enteral Nutrition (ASPEN). Journal of Parenteral and Enteral Nutrition, v. 33, n. 3, pág. 277-316, 2009.

28. Blaser, A. R. et al. Nutrição enteral precoce em pacientes críticos: diretrizes de prática clínica da ESICM. Medicina de terapia intensiva , v. 43, n. 3, pág. 380-398, 2017.

29. Nutrition Therapy in the Patient with COVID-19 Disease Requiring ICU Care, SCCM and ASPEN. Society of Critical Care Medicine, 2020.

30. Singer, P. et al. ESPEN guideline on clinical nutrition in the intensive care unit. Clinical Nutrition, set. 2018.

31. Arkin, N. et al. Nutrition in critically ill patients with COVID-19: Challenges and special considerations. Clinical Nutrition, v. 39, pág. 2327-2328, 202 